\title{
Toxicidad del cobre sobre la morfología de estomas de Gochnatia arequipensis Sandwith (Asteraceae) de dos localidades de Arequipa, Perú
}

\section{Copper toxicity on the stomata morphology of Gochnatia arequipensis Sandwith (Asteraceae) from two localities of Arequipa, Perú}

\author{
David Angel Apaza Machacal ${ }^{1}$, Bertha Roxana Mestas Valdivia ${ }^{1}$, \\ Frey Francisco Romero Vargas ${ }^{1}$, Ronald Demetrio Navarro Oviedo ${ }^{1 *}$
}

\section{RESUMEN}

El exceso de metales pesados, como el cobre, provoca toxicidad en las plantas, en consecuencia, genera cambios en sus tejidos aéreos, afectando sus estomas cuya principal función es el intercambio gaseoso. Sin embargo, la densidad y morfometría de estos estomas podrían variar para compensar el contenido hídrico provocado por los cambios ambientales.

El propósito de este estudio fue determinar el área foliar, la densidad y la morfometria de los estomas de hojas de Gochnatia arequipensis Sandwith (Asteraceae) en relación al contenido de cobre foliar, determinado mediante espectrofotometría de absorción atómica, colectadas en diferentes puntos de muestreo. En esta investigación se realizó un diseño estratificado, para lo cual se recolectaron hojas de la planta en estudio desarrolladas en el distrito de Quequeña a diferentes distancias del relave perteneciente al PAM Kiowa y también en el distrito de Characato.

El área foliar fue menor en plantas de los puntos más próximos al PAM Kiowa $\left(55.8 \pm 11.27 \mathrm{~mm}^{2}\right)$ que el área foliar de las plantas del distrito de Characato $\left(62.72 \pm 6.94 \mathrm{~mm}^{2}\right)$. Por otro lado, la densidad estomática $\left(154.2 \pm 32.9 \mathrm{estomas} / \mathrm{mm}^{2}\right)$ fue mayor en las plantas de los puntos próximos al PAM Kiowa mientras que su área estomática disminuyó $\left(315.02 \pm 31.114 \mu \mathrm{m}^{2}\right)$. La apertura estomática fue mayor en las plantas de Characato $\left(73.04 \pm 8.3 \mu \mathrm{m}^{2}\right)$.

Cuando la cantidad de cobre foliar es mayor, el área foliar sufre una disminución, la densidad estomática disminuye conforme las plantas se alejan del PAM Kiowa.

Palabras claves: densidad estomática, morfometría estomática, pasivo ambiental minero.

\section{ABSTRACT}

The excess of heavy metals, such as copper, causes toxicity in plants, consequently, it generates changes in their aerial tissues, affecting their stomata whose main function is gas exchange. However, the density and morphometry of these stomata could vary to compensate for the water content caused by these environmental changes.

The purpose of this study was to determine the leaf area, density and morphometry of leaf stomas of Gochnatia arequipensis Sandwith (Asteraceae) in relation to leaf copper content, determined by atomic absorption spectrophotometry, collected at different sampling points. In this research, a stratified design was carried out, for which leaves of the study plant developed in the district of Quequeña were collected at different distances from the tailings belonging to the Kiowa PAM and also in the Characato district.

The leaf area was lower in plants from the points closest to the Kiowa PAM $\left(55.8 \pm 11.27 \mathrm{~mm}^{2}\right)$ than the leaf area of the Characato district plants $\left(62.72 \pm 6.94 \mathrm{~mm}^{2}\right)$. On the other hand, the stomatal density $\left(154.2 \pm 32.9\right.$ stomata / $\left.\mathrm{mm}^{2}\right)$ was greater in the plants of the points near the Kiowa PAM while its stomatal area decreased $\left(315.02 \pm 31.114 \mu \mathrm{m}^{2}\right)$. The stomatal opening was greater in the Characato plants $\left(73.04 \pm 8.3 \mu \mathrm{m}^{2}\right)$.

When the amount of leaf copper is greater, the leaf area suffers a decrease, the stomatal density decreases as the plants move away from the Kiowa PAM.

Keywords: stomatal density, stomatal morphometry, mining environmental liabilities.

\footnotetext{
1 Laboratorio de Bioquímica y Biología Molecular, Facultad de Ciências Biológicas, Departamento de Biologia. Universidad Nacional de San Agustín. Arequipa, Perú

* Autor por correspondencia: rnavarroo@unsa.edu.pe
}

Fecha de Recepción: 23 mayo, 2019.

Fecha de Aceptación: 21 septiembre, 2019. 


\section{Introducción}

La disponibilidad de los metales pesados en los suelos depende de procesos naturales o antropológicos como la minería. En Perú, el cobre posee un porcentaje alto de exportaciones al extranjero y Arequipa tiene gran significancia por aportar el 17\% de esta actividad (Vásquez y Zurita, 2016). El presente estudio considera dos zonas no agrícolas donde se desarrolla Gochnatia arequipensis Sandwith: un suelo contaminado por cobre (PAM Kiowa) como consecuencia de la actividad minera $(246,615 \pm 31,44 \mathrm{ug} / \mathrm{g}$ de suelo) y un suelo no contaminado por actividad minera (localidad de Yanayaco, distrito de Characato) $(63,365 \pm 9,23 \mathrm{ug} / \mathrm{g}$ de suelo). Asimismo, se ha reportado que las plantas de Gochnatia arequipensis Sandwith, que crecen en el PAM Kiowa exhiben 161,947 $\pm 2,60$ ug de cobre por gramo de tejido foliar y las plantas de la localidad de Yanayaco presentan 38,943 $\pm 0,17$ ug de cobre por gramo de tejido foliar (Vasquez K.E. 1998, datos no publicados).

Los efectos de la fitotoxicidad del $\mathrm{Cu}$ en plantas son numerosos, tales como clorosis en las hojas, crecimiento es raquítico, desarrollo pobre, ramificaciones reducidas, obstrucción y coloración oscura (Nair y Chung, 2015), disminución del área foliar y cambios en el número y morfometria de los estomas (Panou-Filotheou et al., 2001).

En el presente trabajo el metal pesado $\mathrm{Cu}$ fue elegido por estar involucrado en varios procesos fisiológicos, como la fotosíntesis, la respiración, la distribución de carbohidratos, la reducción y fijación de nitrógeno, el metabolismo de las proteínas, las relaciones hídricas, entre otros (Bussler, 1981; Lanaras et al., 1993; Vinit-Dunand et al., 2002).

Los estomas cumplen un rol de suma importancia en la superficie de las hojas regulando la perdida de agua y captación del dióxido de carbono durante la fotosíntesis. Por tal motivo, la relación de agua y acumulación de biomasa vegetal es influenciada por el funcionamiento de los estomas, ya que tales estructuras se encuentran en las hojas, estos órganos son sensibles a los cambios ambientales, y reflejan alteraciones morfológica como consecuencias del efecto del estrés en el medio (Trewaras, 2003; Sánchez-Diaz y Aguirreola, 2012).

Nuestro trabajo se centra en determinar los cambios provocados por el cobre sobre el área foliar, densidad y morfometria de los estomas, ya que estas variables pueden ayudar a un mejor entendimiento de efecto de este metal pesado sobre la supervivencia de Gochnatia arequipensis Sandwith.

\section{Materiales y Métodos}

\section{Material vegetal y área de estudio}

Los especímenes de Gochnatia arequipensis Sandwith utilizados en el presente trabajo fueron identificados por el Instituto Científico Michael Owen Dillon (IMOD) y por el Herbarium Areqvipense (HUSA); en este último se puede encontrar los especímenes depositados. Las plantas de G. arequipensis Sandwith, del orden Asterales, se colectaron considerando las siguientes características: cobertura vegetal, síntomas de fitotoxicidad y distancias de la mina (Bech et al., 2002). Se realizó un diseño estratificado considerando dos localidades de muestreo: la primera fue Quequeña, en los alrededores del relave del Pasivo Ambiental Minero (PAM) Kiowa donde se eligieron 5 puntos de muestreo (q1, q2, q3, q4 y q5), cada punto con 100 metros de separación el uno del otro. El primer punto de muestreo tuvo las siguientes coordenadas $71^{\circ} 20^{\prime} 64^{\prime \prime} \mathrm{O}$ y $16^{\circ} 32$ '29's (Figura 1). La segunda localidad de muestreo fue el distrito de Characato, considerando 4 puntos de muestreo (c1, c2, c3 y c4), con una separación de 100 metros entre ellos, el primer punto de esta localidad está ubicado a $71^{\circ} 27^{\prime} 15.87^{\prime \prime}$ O y $16^{\circ} 28^{\prime} 37.49^{\prime \prime}$ S. De igual manera, se colectaron muestras de suelo en los que se desarrollan los ejemplares de $G$. arequipensis Sandwith a una profundidad de $0-20 \mathrm{~cm}$ (datos no mostrados).

\section{Determinación de $\mathrm{Cu}$ en tejido foliar}

Tejidos foliares fueron secados a temperatura ambiente durante 2 semanas y luego enviados al Laboratorio de suelos, plantas, aguas y fertilizantes (LASPAF) del Departamento de Agronomía de la Universidad Nacional Agraria La Molina para la determinación de $\mathrm{Cu}$ por el método de espectrofotometría de absorción atómica.

\section{Determinación de densidad estomática}

En campo, las hojas de $G$. arequipensis Sandwith fueron colectadas en solución F.A.A. 


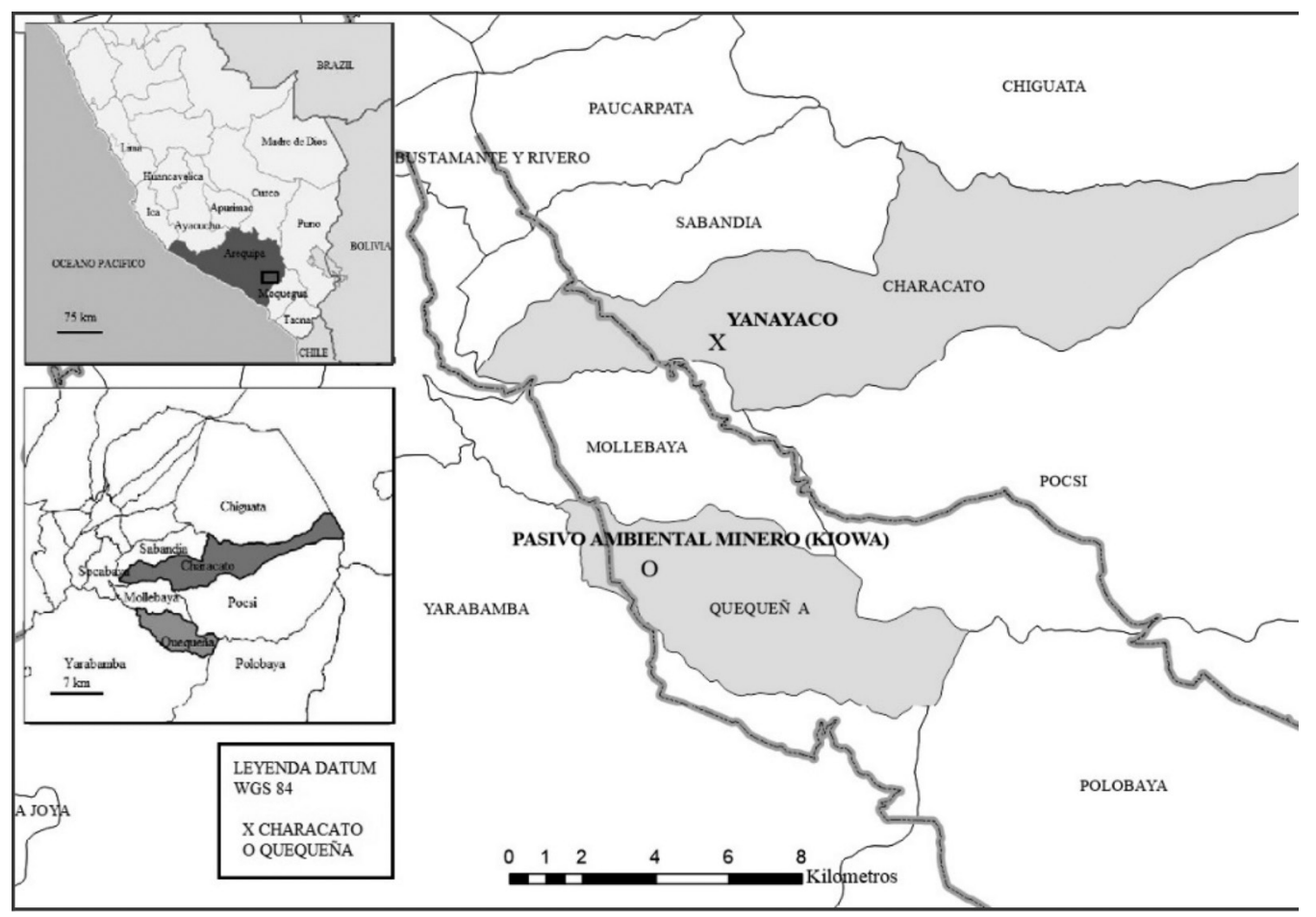

Figura 1. Situación geografía del área de estudio. Se muestra la ubicación de los puntos de muestreo en las proximidades del relave del PAM Kiowa (X) y el distrito de Characato de la localidad de Yanayaco (O). WGS 84= World Geodetic System 1984.

(850 ml de alcohol etílico al 70\%, $100 \mathrm{ml}$ de formaldehído al $40 \%$ y $50 \mathrm{ml}$ de ácido acético glacial). Después de 24 horas, las hojas fueron lavadas con agua destilada y sumergidas en lejía comercial bajo agitación mecánica durante 20 horas. Posteriormente, las hojas fueron preservadas en alcohol etílico al $75 \%$ hasta el montaje de las láminas. Finalmente, se utilizó hojas de afeitar para desprender la epidermis, se coloreó con safranina y los fragmentos de epidermis fueron fijados en solución de agua: glicerina (1:1) (Salas et al., 2001). El conteo de estomas se realizó con un microscopio óptico (PrimoStarZeiss) con objetivo de 40X; se evaluaron 9 campos por muestra y el número de estomas se expresa por $\mathrm{mm}^{2}$ (Domínguez et al., 2014).

\section{Morfometría de estomas y de ostiolos}

Las observaciones fueron realizadas en un microscopio óptico binocular Motic ${ }^{\circledR}$ (objetivo 40X), provisto de una cámara Moticam ${ }^{\circledR}$ BTU y las imágenes fueron analizadas haciendo uso del software MOTIC IMAGES Plus 3.0. Con los datos obtenidos en el paso anterior se procedió a calcular el área de estomas $\left(\mu \mathrm{m}^{2}\right)$ y ostiolos mediante la siguiente formula (Flórez y Cruz, 2004):

$$
\mathrm{A}=(\operatorname{largo} \mathrm{x} \text { ancho } \mathrm{x} \pi)
$$

\section{Determinación del área foliar}

Se seleccionaron hojas con síntomas de fitotoxicidad, las cuales fueron fotografiadas con una cámara Cyber-Short. Teniendo una medida de referencia, se procedió al análisis con el software ImageJ (versión 1.46) para determinar el área en $\mathrm{mm}^{2}$ (Rincón et al., 2012).

\section{Análisis Estadístico}

Para la comparación de los diferentes aspectos analizados se utilizó el estadístico ANOVA y para la comparación de medias se utilizó la prueba de Duncan con un nivel de significancia del 5\% haciendo uso del software S.P.P.S. v. 12. 


\section{Resultados y Discusión}

El análisis por espectrofotometría de absorción atómica, indica que la cantidad promedio de $\mathrm{Cu}$ en el tejido foliar de plantas de G. arequipensis Sandwith provenientes del PAM Kiowa $(78,3 \pm 18,6$ ppm $\mathrm{Cu}$ ) es mayor que en las plantas provenientes de Characato $(72,6 \pm 7,3 \mathrm{ppm}$ de $\mathrm{Cu})$. El ANOVA indica que existe diferencias significativas $(\mathrm{p}<0,05)$ entre los puntos de muestreo. La prueba de comparación de Duncan nos muestra diferencias entre el punto más próximo al PAM Kiowa $(108 \pm 0,71$ $81 \pm 4,24$ ppm de $\mathrm{Cu}$ ) y los puntos más alejados de este PAM (59,5 $\pm 4,9 \mathrm{ppm}$ de $\mathrm{Cu})$, mientras que las cantidades de cobre en los puntos de muestreo del distrito de Characato no muestran variación significativa $(67,5 \pm 3,54$ a 77,0 $\pm 2,83 \mathrm{ppm}$ de $\mathrm{Cu})$ (Tabla 1). La literatura revisada no reporta otros trabajos con la planta en estudio; sin embargo, hay reportes de otras plantas de la misma familia desarrolladas en suelos contaminados por la actividad minera como la de Turmalina (Piura), para plantas de Bidens cynapiifolia $(620 \pm 120 \mathrm{ug}$ $\mathrm{Cu} / \mathrm{g}$ de materia fresca de raíz) (Bech et al., 1997). El hecho de que las plantas de G. arequipensis Sandwith, tengan la capacidad de absorber hasta $108 \pm 0,71 \mathrm{ppm}$ de $\mathrm{Cu}$, a nivel foliar, el cual está por encima de los niveles considerados tóxicos para las plantas, indica que G. arequipensis Sandwith podría ser considerada una planta tolerante a este metal (Kabatas-Pendias y Pendias, 2000). Por estas razones se hace necesario estudios adicionales para corroborar el comportamiento de esta planta frente al cobre.

\section{Área foliar}

El análisis del área foliar de Gochnatia arequipensis Sandwith, indica una disminución que es congruente con la mayor cantidad de $\mathrm{Cu}$ en sus tejidos foliares, lo cual se percibe en los valores promedio: $55,80 \pm 11,278 \mathrm{~mm}^{2}$ y $62,72 \pm 6,946 \mathrm{~mm}^{2}$, para la localidad del PAM Kiowa y Characato, respectivamente. El ANOVA indica diferencias altamente significativas $(\mathrm{p}<0,01)$ (Figura 2 y Figura 3). La comparación de Duncan indica que el área foliar de las plantas de los puntos más próximos al PAM Kiowa $(44,61 \pm 6,61,50 \pm 6,2$ y $50,76 \pm 5,9 \mathrm{~mm}^{2}$ ) tienen una menor área foliar, mientras que en las plantas de Characato el área es mayor y uniforme $\left(60,07 \pm 5.5\right.$ a $\left.65,24 \pm 8,2 \mathrm{~mm}^{2}\right)$.
Tabla 1. Niveles de Cu foliar en plantas de Gochnatia arequipensis Sandwith procedentes de los diferentes puntos de muestreo del PAM Kiowa y de Characato.

\begin{tabular}{lcc}
\hline Localidad & Puntos de muestreo & $\begin{array}{c}\text { Cu en el tejido } \\
\text { foliar (ppm) }\end{array}$ \\
\hline \multirow{2}{*}{ PAM } & q1 & $108,5 \pm 0,707$ \\
Kiowa & q2 & $81,4 \pm 0,242$ \\
& q3 & $77,5 \pm 12,021$ \\
& q4 & $65 \pm 5,657$ \\
Characato & q5 1 & $59,5 \pm 4,95$ \\
& c2 & $77 \pm 2,83$ \\
& c3 4 & $75 \pm 15,556$ \\
& c4 & $71 \pm 2,828$ \\
\hline
\end{tabular}

\begin{tabular}{cc}
\hline Significancia & $\mathrm{p}<0,05$ \\
\hline
\end{tabular}

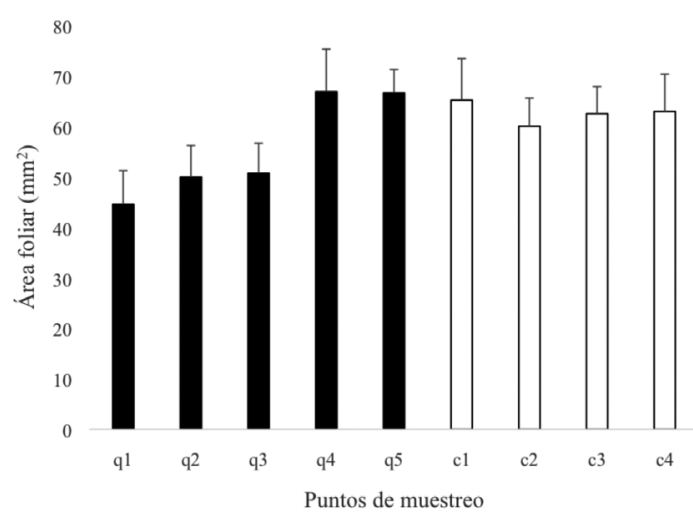

Figura 2. Variaciones del área foliar $\left(\mathrm{mm}^{2}\right)$ de Gochnatia arequipensis Sandwith en relación a la disponibilidad de $\mathrm{Cu}$ en los puntos de muestreo del PAM Kiowa (q1 - q5) y de Characato (c1 - c4). Los histogramas muestran los valores promedio de 45 repeticiones.

La disminución del área foliar en las plantas tratadas con $\mathrm{Cu}$ probablemente afecta el fenómeno de transpiración (Lanaras et al., 1993), lo cual es observado el plantas tratadas con otros metales pesados (Barceló et al., 1988). Nuestros resultados indican que el $\mathrm{Cu}$ provoca estrés a la planta y esta toma medidas para tolerar el exceso en su tejido foliar, como incremento en la expresión de la enzima POD y disminución de los niveles de prolina (datos no mostrados). Este costo de tolerancia está documentado por varios estudios que reportan una pérdida de biomasa, y crecimiento lento en comparación con otros especímenes no expuestos a metales pesados (Panou - Filotheou et al., 2001; Ruiz y Armienta, 2012). La pérdida de biomasa y el crecimiento lento probablemente se debe a 


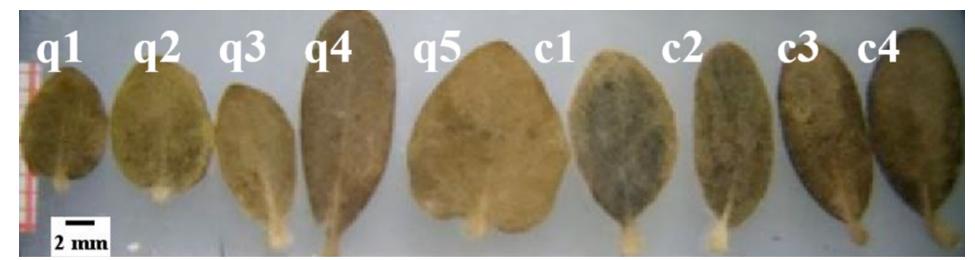

Figura 3. Imágenes que muestran la superficie de hojas de Gochnatia arequipensis Sandwith colectadas en los puntos de muestreo del PAM Kiowa (q1-q5) y de Characato (c1-c4).

que el $\mathrm{Cu}$ afecta el proceso de expansión celular provocando la reducción del tamaño celular y en consecuencia reducción del tamaño de la raíz, del tallo y de las hojas (Kasim, 2006). PanouFilotheou et al., (2001) y Vinit-Dunand et al., (2002), indican que concentraciones crecientes de $\mathrm{Cu}$ alteran los parámetros estructurales de la hoja como disminución en el tamaño, clorosis y engrosamiento de la lámina foliar.

\section{Densidad y morfometría de estomas y ostiolos}

Los especímenes analizados al ser de un ambiente xerofítico, solo presentaban estomas en el envés de las hojas. La densidad estomática media varía entre $154,2 \pm 32,9$ y $115,5 \pm 10,1$ estomas/ $\mathrm{mm}^{2}$ en las localidades de PAM Kiowa y Characato, respectivamente. El ANOVA revela que no hay diferencias significativas ( $p>0,05)$ en los puntos de muestreo (Tabla 2). Sin embargo, la densidad estomática es mayor en los puntos de muestreo más cercanos al PAM Kiowa $(154,3 \pm 29,7$ estomas/ $\mathrm{mm}^{2}$ ) en comparación a la media de Characato $\left(115,5 \pm 10,1\right.$ estomas $\left./ \mathrm{mm}^{2}\right)$ (Figura 4 y Tabla 2 ). Las estomas del PAM Kiowa tuvieron un largo de $22,42 \pm 1,2 \mu \mathrm{m}$ y un ancho de $17,92 \pm 0,95 \mu \mathrm{m}$ y los estomas de las plantas de Characato tuvieron un largo de $23,83 \pm 0,98 \mu \mathrm{m}$ y un ancho de $18,85 \pm 0,7$ $\mu \mathrm{m}$. Mientras que los ostiolos presentan valores promedios ligeramente menores (largo: 22,42 $\pm 1,2$ $\mu \mathrm{m}$ y ancho: $17,92 \pm 0,95 \mu \mathrm{m})$ en el PAM Kiowa en relación a los presentados por Characato (largo: $23,83 \pm 0,98 \mu \mathrm{m}$ y ancho: $18,85 \pm 0,7 \mu \mathrm{m})$.

El análisis de varianza (Tabla 2) muestra que no existen diferencias significativas $(p>0,05)$ entre el largo y ancho de los estomas frente al largo del ostiolo. Sin embargo, el ANOVA revela que el ancho del ostiolo, si muestra diferencias significativas $(\mathrm{p}<0,01)$.

Con respecto al área de los estomas de las plantas del PAM Kiowa se encontró un promedio de $315,02 \pm 31,114 \mu \mathrm{m}^{2}$ y para las plantas de Characato un promedio de $352,54 \pm 17,572 \mu \mathrm{m}^{2}$

Tabla 2. Valores promedio y desviaciones típicas de densidad estomática y morfometría de estomas y ostiolos de Gochnatia arequipensis Sandwith.

\begin{tabular}{lcccccccc}
\hline \multicolumn{10}{c}{ Variables } \\
\hline Localidad & $\begin{array}{c}\text { Puntos } \\
\text { de } \\
\text { muestreo }\end{array}$ & $\begin{array}{c}\text { Densidad } \\
\text { estomática } \\
\left(\mathrm{mm}^{2}\right)\end{array}$ & $\begin{array}{c}\text { Longitud } \\
\text { del estoma } \\
(\mu \mathrm{m})\end{array}$ & $\begin{array}{c}\text { Ancho } \\
\text { del estoma } \\
(\mu \mathrm{m})\end{array}$ & $\begin{array}{c}\text { Longitud } \\
\text { del ostiolo } \\
(\mu \mathrm{m})\end{array}$ & $\begin{array}{c}\text { Ancho } \\
\text { del ostiolo } \\
(\mu \mathrm{m})\end{array}$ & $\begin{array}{c}\text { Área } \\
\text { estomática } \\
\left(\mu \mathrm{m}^{2}\right)\end{array}$ & $\begin{array}{c}\text { Área } \\
\text { del ostiolo } \\
\left(\mu \mathrm{m}^{2}\right)\end{array}$ \\
\hline \multirow{6}{*}{ PAM } & $\mathrm{q} 1$ & $154,333 \pm 29,74$ & $21,54 \pm 0,45$ & $17,43 \pm 0,45$ & $12,77 \pm 0,53$ & $7,16 \pm 0,48$ & $294,84 \pm 11,71$ & $71,98 \pm 7,83 \mathrm{~b}$ \\
Kiowa & $\mathrm{q} 2$ & $146,333 \pm 40,01$ & $21,42 \pm 0,11$ & $17,95 \pm 0,93$ & $12,35 \pm 0,22$ & $6,87 \pm 0,14$ & $302,01 \pm 15,36$ & $66,64 \pm 0,69$ \\
& $\mathrm{q} 3$ & $155 \pm 47,44$ & $23,04 \pm 1,85$ & $18,97 \pm 1,07$ & $14,39 \pm 1,83$ & $6,94 \pm 0,53$ & $344,28 \pm 46,96$ & $78,02 \pm 5,80$ \\
& $\mathrm{q} 4$ & $148,667 \pm 42,82$ & $23,61 \pm 1,02$ & $18,21 \pm 0,82$ & $12,90 \pm 1,44$ & $6,82 \pm 0,36$ & $338,16 \pm 29,38$ & $69,28 \pm 10,47$ \\
& $\mathrm{q} 5$ & $166,667 \pm 25,38$ & $22,49 \pm 0,38$ & $17,03 \pm 0,24$ & $11,75 \pm 0,83$ & $5,91 \pm 0,41$ & $300,82 \pm 9,31$ & $54,37 \pm 2,93$ \\
\hline \multirow{2}{*}{ Characato } & $\mathrm{c} 1$ & $116 \pm 12,49$ & $23,09 \pm 0,35$ & $18,40 \pm 0,72$ & $13,35 \pm 0,31$ & $7,18 \pm 0,29$ & $333,53 \pm 8,65$ & $75,32 \pm 3,89$ \\
& $\mathrm{c} 2$ & $118 \pm 13,22$ & $23,24 \pm 0,54$ & $19,64 \pm 0,64$ & $13,39 \pm 0,65$ & $7,80 \pm 0,14$ & $358,74 \pm 20,24$ & $81,99 \pm 2,95$ \\
& $\mathrm{c} 3$ & $112,667 \pm 8,02$ & $23,81 \pm 0,37$ & $19,0 \pm 0,23$ & $12,81 \pm 0,51$ & $7,11 \pm 0,64$ & $355,36 \pm 10,12$ & $71,70 \pm 9,06$ \\
\multicolumn{2}{c}{ Significancia } & $115,667 \pm 11,59$ & $25,14 \pm 0,68$ & $18,36 \pm 0,44$ & $12,75 \pm 0,17$ & $6,30 \pm 0,11$ & $362,54 \pm 18,67$ & $63,12 \pm 1,62$ \\
\hline
\end{tabular}




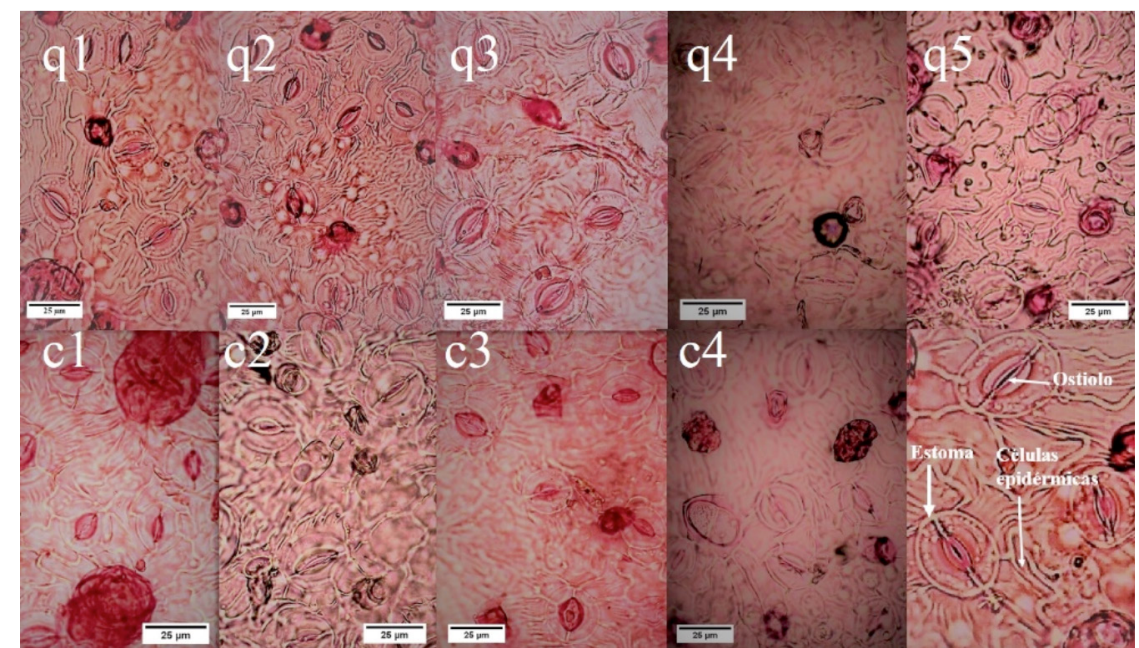

Figura 4. Vista frontal de la epidermis de Gochnatia arequipensis Sandwith colectadas en el PAM Kiowa (q1-q5) y en la localidad de Characato (c1-c4). Se muestra las dimensiones y área de estomas y ostiolos de plantas desarrolladas en ambas áreas de estudio. Las fotografías fueron tomadas con un aumento de 40X. Barra $=25 \mu \mathrm{m}$.

con diferencias significativas $(\mathrm{p}<0,01)$. Mientras que el área del ostiolo presenta medias de $68,06 \pm 9,799 \mu \mathrm{m}^{2}$ y $73,04 \pm 8,387 \mu \mathrm{m}^{2}$ en el PAM Kiowa y Characato, respectivamente con diferencias altamente significativas $(\mathrm{p}<0,01)$ (Figura 4 y Tabla 2).

La densidad estomática observada en las plantas de Gochnatia arequipensis Sandwith (hipostomatica) indica que existe una mínima diferencia en relación a los puntos de muestreo. Si bien, la densidad estomática es ligeramente mayor en las hojas de las plantas provenientes de los puntos muestreo del PAM Kiowa (con mayor contenido de $\mathrm{Cu}$ foliar), su área es menor. Esta menor área y mayor densidad estomática, es una alteración estructural provocada por las concentraciones altas de $\mathrm{Cu}$ (Panou-Filotheou et al., 2001), que le permiten a la planta equilibrar la cantidad de agua perdida por transpiración a causa de una menor área foliar. Por el contrario, el tamaño y densidad estomática se mantienen constantes en las plantas provenientes de los puntos de muestreo de Characato y los puntos más alejados de PAM Kiowa. Estas afirmaciones se basan en que los metales pesados inhiben el alargamiento celular debido a una pérdida de turgencia, a causa de la fuga de constituyentes celulares (Wainwright y Woolhouse, 1977), ya que podrían afectar el desarrollo y función de los estomas (Moustakas et al., 1997). Sin embargo, el efecto de metales pesados depende de la especie vegetal, pues los metales pesados como el cadmio provocan un aumento de la densidad estomática y una disminución de la transpiración en plantas de Phaseolus vulgaris debido al cierre del ostiolo (Barceló et al., 1988), por el contrario en plantas de Fagus sylvatica provoca un aumento de la transpiración (Hagemeyer et al., 1986). Asimismo, se ha encontrado que concentraciones de cobre por encima del umbral de toxicidad provocan alteraciones morfológicas y anomalías en el desarrollo de tricomas y estomas, disminución del tamaño y aumento del número de estomas (Kastori et al., 1992). Además, se ha observado que en plantas que excluyen el cobre, la densidad de estomas es menor que en plantas acumuladoras de cobre (Fu et al., 2015). El exceso de $\mathrm{Cu}$ puede influir en la distribución de iones calcio, magnesio y potasio de la raíz a las hojas; este último ion influye en la turgencia y expansión de las hojas (Alaoui-Sossé et al., 2004), ya que es un constituyente importante en el movimiento iones durante el proceso de apertura y cierre estomático (Sánchez-Díaz y Aguirreolea, 2012).

\section{Conclusiones}

El área foliar fue menor en plantas de los puntos más próximos al PAM Kiowa que en plantas del distrito de Characato. Por otro lado, la densidad estomática $\left(154,2 \pm 32,9\right.$ estomas $\left./ \mathrm{mm}^{2}\right)$ 
fue mayor en las plantas de los puntos próximos al PAM Kiowa mientras que su área estomática disminuyó. La apertura estomática fue mayor en las plantas provenientes de suelos con bajo contenido de $\mathrm{Cu}$ (Characato).
Cuando la cantidad de cobre foliar es mayor, el área foliar sufre una disminución, por el contrario la densidad estomática disminuye conforme lo hace los niveles de $\mathrm{Cu}$ en suelo (PAM Kiowa).

\section{Literatura Citada}

Alaoui-Sossé, B.; Genet, P.; Vinit-Dunand, F.; Tossaint. M.; Epron, D.; Badon, P.

2004. Effect of copper on growth in cucumber plants (Cucumis sativus) and its relationships with carbohydrate accumulation and changes in ion contents. Plant Science, 166 (5): 1213-1218.

Barceló, J.; Vazquez, M.; Poschenrieder, C.

1988. Structural and ultra structural disorders in cambiumtreated bush bean plants (Phaseolus vulgaris L). New Phytol, 108 (1): 37-49.

Bech, J.; Poschenrieder, C.; Barceló, J.; Lansac, A. 2002. Plants from mine spoils in the South American Area as Potential Sources of Germplas, for Phytoremediation Technologies. Engineering: in life sciences. Acta Biotechnol, 22 (1-2): 5-11.

Bech, J.; Poschenrieder, C.; Llugany, M.; Barceló, J.; Tume, P.; Tobias, F.; Barranzuela, J.; Vásquez,E. 1997. Arsenic and heavy metal contamination of soil and vegetation around a copper mine in Northern Peru. The Science of the Total Environment, 203 (1): 83-91.

Bech, J.; Roca, N.; Tume, P.; Ramos-Miras, J.; Gil, C.; Boluda, R. 2016. Screening for new accumulator plants in potential hazards elements polluted soil surrounding Peruvian mine tailings. J. Catena, 136: 66-73.

Bussler, W.

1981. Physiological functions and utilization of copper. In: Lonegaran, J.F.; Robson, A.D.; Graham, R.D.; (Eds). Copper in soils and plants. Academic Press. New York,US. pp. 213-234 .

Domínguez, A.; Pérez, Y.; Alemán, S.; Sosa, M.; Fuentes, L.;

Darias, R.; Demey, J.; Rea, R.; Sosa, D.

2014. Respuesta de cultivares de Phaseolus vulgaris L. al estrés por sequía. Biotecnología Vegetal, 14 (1): 26-36.

Flórez, V.; Cruz, R. 2004. Guía de laboratorio de Fisiología. UNIBIBLOS. Bogotá, Colombia. 57 p.

Fu, L.; Chen, C.; Wang, B.; Zhou, X.; Li, S.; Guo, P.; Shen, Z. 2015. Differences in copper absorption accumulation between copper-exclusion and copper-enrichment plant: a comparison of structure and physiological responses. PLOS ONE, 1-18.

Hagemeyer, J.; Kahle, H.; Breckle, S.W.; Waisel, Y. 1986. Cadmium in Fagus sylvatica L. trees and seedlings: Leaching, uptake and interconnection with transpiration. Water, Air, and Soil Pollution, 26 (4): 347-359.

Kabatas-Pendias, A.; Pendias, $\mathrm{H}$.

2001. Trace Elements in Soils and Plant. CRC Press. Boca Raton, Florida, US. 412 p.

Kasim, W.

2006. Changes Induced by Copper and Cadmium Stress in the Anatomy and Grain Yield of Sorghum bicolor (L.) Moench. International Journal of Agriculture \& Biology, 8: 123-128.
Kastori, R.; Petrovic, M.; Petrovic, N.

1992. Effect of excess lead, cadmium, copper, and zinc on water relations in sunflower. Plant Nutrition, 15: 2427-2439.

Lanaras, T.; Moustakas, M.; Symeonidis, L.; Diamantoglou, S.; Karataglis, S.

1993. Plant metal content, growth responses and some photosynthetic measurements on field-cultivated wheat growing on ore bodies enriched in $\mathrm{Cu} .88$ (2): 307-314.

Moustakas, M.: Ouzonidou, G.; Symeonidis, L.; Karataglis, S. 1996. Field study of the effects of excess copper on wheat photosynthesis and productivity. 43 (3): 531-539

Nair, P.; Chung, I.

2015. Study on the correlation between copper oxide nanoparticles induced growth suppression and enhanced lignification in Indian mustard (L.), 113: 302-313

Vásquez, A.; Zurita, V.

2016. Año 5 - Número 6. Gerencia de Políticas y Análisis Económico. OSINERGMIN. Perú. 22 p.

Panou-Filotheou, H.; Bosabalidis, A.M.; Karatagis, S.

2001. Effects of copper toxicity on leaves of oregano (sudsp. hirtum), 166: 1497-1504.

Rincón, G.; Olarte, M.A.; Pérez, J.C.

2012. Determinación del Área Foliar en Fotografías Tomadas con una Cámara Web, un Teléfono Celular o una Cámara Semiprofesional. Rev. Fac. Nal. Agr. Medellín, 65 (1): 6399-6405.

Ruiz, E.; Armienta, M.

2012. Acumulación de arsénico y metales pesados en maíz en suelos cercanos a jales o residuos mineros. Rev. Int. Contam. Ambie, 28 (2): 103-117.

Salas, J.; Sanabria, M.; Pire, R.

2001. Variación en el índice y densidad estomática en las plantas de tomate (Lycopersicum esculentum Mill.) sometidas a tratamientos salinos. Bioagro, 13(3): 99-104.

Sánchez-Díaz, M.; Aguirreolea, J.

2012. Transpiración y control estomático. In AzcónBieto, J.; Talón, M.; (eds). Fundamentos de la fisiología vegetal. McGraw-Hill Interamericana. Madrid, España. 41-56 pp.

Trewaras, A. 2003. Aspects of Plant Intelligence. Annals of Botany, 92(1): 1-20.

Vinit-Dunand, F.; Epron, D.; Alaoui-Sosse, B.; Badot, P.M. 2002. Effects of copper on growth and on photosynthesis of mature and expanding leaves in cucumber plants., 163: 53-58.

Wainwright, S.; Woolhouse, H.W.

1977. Some Physiological Aspects of Copper and Zinc Tolerance in Agrostis tenuis Sibth: Cell Elongation and Membrane Damage., 28(4): 1029-1036. 
